\title{
Analysis of the effect of BER and Q-factor on free space optical communication system using diverse wavelength technique
}

\author{
A. A.Anis ${ }^{1, *}$, C.B. M. Rashidi ${ }^{1}$, A. K. Rahman ${ }^{2}$, S. A. Aljunid ${ }^{1}$, and N. Ali ${ }^{1,3}$
}

${ }^{1}$ Advanced Communication Engineering, Centre of Excellence - School of Computer \& Communication Eng, Universiti Malaysia Perlis, (UniMAP) Kangar, 01000, Kangar, Perlis

${ }^{2}$ Department of Electrical and Electronic Eng, Universiti Malaysia Sarawak, (UNIMAS), Sarawak

${ }^{3}$ Semiconductor Photonics \& Integrated Lightwave Systems (SPILS), School of Microelectronic Engineering, Universiti Malaysia Perlis, Pauh Putra, 02600 Arau, Perlis, Malaysia

\begin{abstract}
This paper presents the analysis of the effect of BER and Q-factor on free space optical communication system using the diverse wavelength technique. The visibility is gradually decreased due to attenuation effects such as weather conditions as the bit error rate increases. Despite this disadvantage, FSO performance could be further improved by using a wide range of wavelengths of the laser beam. The results show good performance for $1550 \mathrm{~nm}$ wavelength, where the wavelength can accommodate visibility for 6 $\mathrm{km}$ at link range of $1 \mathrm{~km}$, and Q-factor 25 at the system performance BER $=10^{-124}$ could improve the system by $67 \%$ compared to $850 \mathrm{~nm}$ and $785 \mathrm{~nm}$ wavelength, which could enhance the system by only $2 \%$. This clearly show that the superior capabilities of $1550 \mathrm{~nm}$ wavelength in handling attenuation problems and capability to reduce the power loss compared to $850 \mathrm{~nm}$ and $785 \mathrm{~nm}$ wavelength.
\end{abstract}

\section{Introduction}

Free Space Optics (FSO) has ultimately become a major leading topic in communication systems nowadays. It is a new technology that utilizes a light beam propagating through the atmosphere. The signal transmission is from the transmitter to transfer data received on the other side of the two point communication system. The FSO communication is also called as Optical Wireless Communication, Fibreless, or Laser Communication [1].

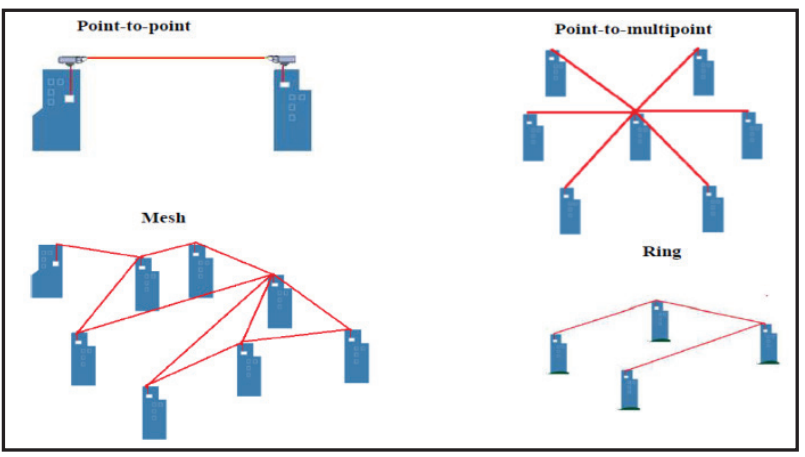

Fig. 1. Terrestrial FSO links [2]

Fig. 1 shows the types of conventional terrestrial FSO links. The high carrier frequency of FSO in the range of $20 \mathrm{THz}$ to $375 \mathrm{THz}$ enables it to transfer data at really high rates. As FSO is offering a wide broadband service, it needs Line of Sight (LOS) communication between the transmitter and receiver [3]. It is due to the principle of light which travels in straight line. It can be implemented as the other choice for an Optical Fiber replacement. Modulation from a laser beam is used to transmit optical binary information. Modulation bandwidth of FSO is better than the optical fiber communication as the FSO system is capable to transfer more data at a time with faster rate. The output modulated optical signal transmits through the atmosphere from a transmitter to a receiver [4]. Fig. 2 demonstrates the transmitter and receiver of a FSO system. Both the transmitter and the receiver is connected by the atmosphere as the transmission medium of the optical signals.

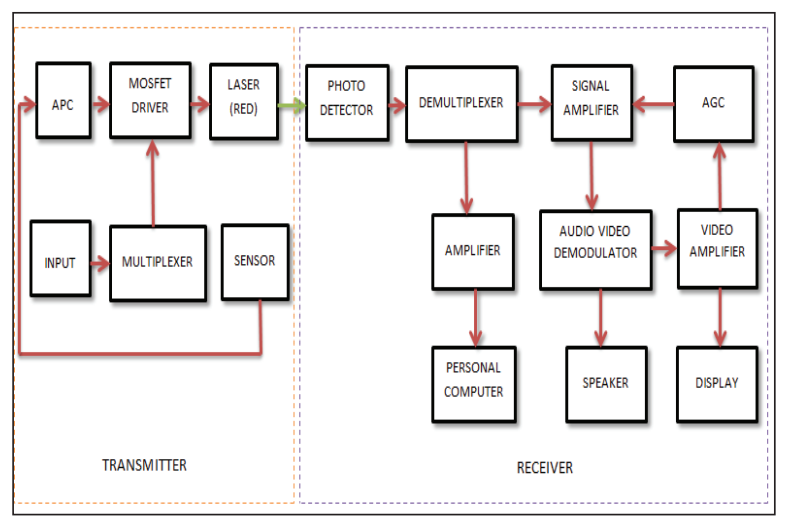

Fig. 2. The primary parts of a FSO system. FSO must consist of a transmitter and a receiver.

\footnotetext{
* Corresponding author: anis afina@ymail.com
} 
There are various FSO employed situations lately under research. The examples are ground-to-ground, satellite uplink/downlink, inter-satellite or deep space probes to ground, ground-to-air e.g. UAV, HAP / air-toground terminal [5]. The increased in the application number of wireless communication have many disadvantages. Such drawbacks are limitation of power, bandwidth regulations, high data rates etc. Nonetheless, FSO has various benefits to be shown. They are no licensing requirements for link utilization, absence of radio frequency radiation hazards, no need of road digging as in the case of optical fiber, wider bandwidths, and low power consumption [6-8]. FSO is affected by attenuation of the atmosphere caused by the unsteady weather conditions such as haze, rain, and fog. These natural phenomena cause attenuation in the transmitted signal. This leads to high bit error rate or signal loss at the receiver end [2]. When a laser beam transmits via the atmosphere, it experiences power loss caused by multiple known factors. One of them is absorption and scattering loss as mentioned in Beer's Law [9].

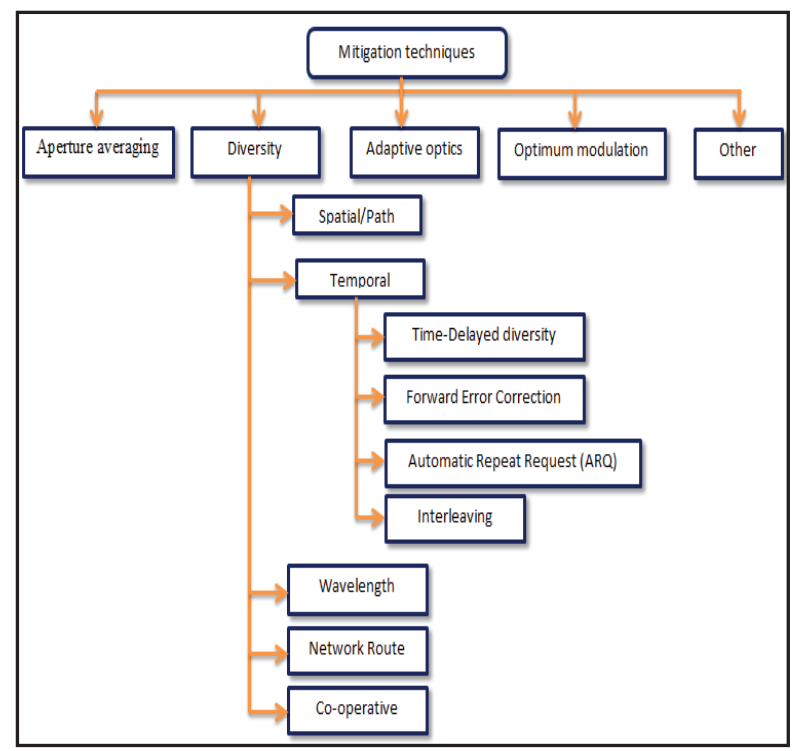

Fig. 3. Tree diagrams showing the various atmospheric mitigation techniques.

The photon of the beam bouncing with haze particles in the mid-air caused a condition called as the absorption. It caused the link margin, distance and the availability of the link to reduce greatly [1]. Scattering is the scattering of a beam of transmission in a range of directions as an impact of physical interactions. When a particle interrupts an electromagnetic wave, some of the energy of the wave is scattered by the particle and reradiated into a solid angle centered on it. The scattered light is polarized. It is also on the same wavelength as the incident wavelength. Thus there is no loss of energy to the particle [10]. All of these atmospheric conditions act to attenuate the light. They also could block the light path in the atmosphere. The performance of FSO links can be analyzed by prediction of the attenuation factor in terms of visibility. Therefore, employment of FSO requests a thorough and detailed study of local weather patterns. Fig. 3 above shows the tree diagram of various atmospheric mitigation techniques to overcome the limitation of FSO. The study is focused more on the effects of haze on the FSO systems. Weather conditions are to be studied in detail before installing FSO systems. This is to reduce the effects of the atmosphere, and also to ensure that the transmitted power is sufficient and minimal losses during bad weather. The performance of FSO system can be evaluated in many ways, such as by analyzing the BER and Q-factor [11]. These two parameters are closely related and they will be explained more in the budgets link section.

\subsection{Optical Wavelength Approach}

\subsubsection{0-850 $\mathrm{nm}$ Wavelength}

These wavelengths are well suited for FSO operations. Approximately $850 \mathrm{~nm}$, reliable, inexpensive, highperformance transmitter and detector components are readily available. The wavelength is normally used in network and transmission equipment. Highly sensitive silicon (Si) avalanche photodiode (APD) detector technology and advanced vertical-cavity surfaceemitting laser (VCSEL) technology can be employed to operate in this wavelength.

\subsubsection{0-1600 Wavelength}

These wavelengths are suitable for free-space transmission. Nevertheless, the components are generally costly. The detectors are less sensitive and have a smaller receive surface area when compared with Si APD detectors that work in the $850 \mathrm{~nm}$ wavelength. Lastly, 50-65 times as much power can be transferred in $1520-1600 \mathrm{~nm}$ than can be transferred at $780-850 \mathrm{~nm}$ for the same eye safety classification.

\subsection{Essential of Free Space Optic Link Budget}

The haze on FSO systems can be discovered by determining the scattering coefficient. The scattering coefficient is directly affected by the visibility conditions. The low visibility means the concentration and size of the particles are more compared to average visibility. Thus, scattering and attenuation may be triggered easier in low visibility conditions. The atmospheric attenuation can be calculated based on the scattering coefficient. The attenuation coefficient has contributions from the absorption and scattering of laser photons by different aerosols and gaseous molecule in the atmosphere. Since the laserbit wavelengths used (typically $785 \mathrm{~nm}, 850 \mathrm{~nm}$ and $1550 \mathrm{~nm}$ ) were chosen, and they fall inside the transmission windows within the atmospheric absorption spectra, the contributions of absorption to the total attenuation coefficient were found to be very small [12]. Thus, the effects of scattering dominate the total attenuation coefficient. The type of scattering is determined by the size of particular atmospheric particle with respect to the transmission laser wavelength. The scattering coefficient can be expressed as a function of the visibility and wavelength. 
Theoretically, high scattering coefficient caused less visibility due to higher concentration and bigger particle sizes of haze along the path of laser beam. Equation (1) is used to determine the scattering coefficient in hazy days.

$$
\beta=\frac{3.91}{V}\left(\frac{\lambda}{550 n m}\right)^{-q}
$$

where :

$\mathrm{V}=$ visibility in kilometers,

$\lambda=$ wavelength in nanometers

$\mathrm{q}=$ the size distribution of the scattering particles

$\mathrm{q}= \begin{cases}1.6 & \text { for } \mathrm{V}>50 \mathrm{Km} \\ 1.3 & \text { for } 6 \mathrm{Km}<\mathrm{V}<50 \mathrm{Km} \\ 0.585 \mathrm{~V}^{1 / 3} & \text { for } \mathrm{V}<6 \mathrm{Km}\end{cases}$

The visibility depends on several parameters. They are the degree of coherence of the source, on the difference in length between the paths as well as on the detector location with respect to the source. The effectiveness and availability of FSO systems is reduced by low visibility. It can occur during a specific time period within a year or at specific times of the day. Low visibility is termed when the concentration and size of the particles are higher compared to average visibility. Hence, the more scattering and attenuation may cause the low visibility conditions [13]. The following Beer's Law equation (2) is derived to describe the atmospheric attenuation (5) :

$$
\tau(R)=-\frac{P(R)}{P(O)}=e^{-(\beta) R}
$$

where

$\tau(\mathrm{R})=$ transmittance at range $\mathrm{R}$,

$\mathrm{P}(\mathrm{R})=$ laser power at $\mathrm{R}$,

$\mathrm{P}(0)=$ laser power at the source, and

$\beta=$ attenuation (per unit length)

The conversion of Equation (2) can be done as it is in linear scale, in logarithms scale :

$$
\begin{aligned}
\tau(R) & =-10 \log \frac{P(R)}{P(O)} \\
& =10 \log e^{\beta R} \\
& =-10 \log e^{-\beta R}
\end{aligned}
$$

The equations to calculate the atmospheric attenuation on hazy days can be obtained by substituting Equation (1) into (4).

$$
\tau(R)=\log e^{\left[\left(\frac{3.91}{V}\right) *\left(\frac{\lambda}{550 n m}\right)^{-q} * R\right]} d B
$$

The total of received power is calculated using Equation (6):

$$
\mathrm{P}_{\text {received }}=P_{\text {transmit }} \frac{A_{\text {receiver }}}{(\theta R)^{2}} e^{-\alpha R}
$$

$$
\begin{array}{ll}
\mathrm{P}_{\text {transmit }} & =\text { transmitted power }(\mathrm{Watt}) \\
\mathrm{A}_{\text {receiver }} & =\text { area of the receiver }\left(\mathrm{m}^{2}\right) \\
\theta & =\text { beam divergence in radian }(\mathrm{rad}) \\
\mathrm{R} & =\text { distance between the transceiver }(\mathrm{m}) \\
\alpha & =\text { average atmospheric attenuation coefficient } \\
(\mathrm{dB} / \mathrm{km}) &
\end{array}
$$

The total of received power could be compared to the total of power transmitted and the area of the collection aperture. It is inversely proportional to the square of the beam divergence and the square of the link range.

Table 1 shows the specifications of FSO system used in this paper. Data calculations and simulations are also made using the specifications set.

Table 1. FSO Evaluation parameters.

\begin{tabular}{|c|c|}
\hline Parameter & Value \\
\hline $\begin{array}{c}\text { Transmission } \\
\text { power }\end{array}$ & $70 \mathrm{~mW}$ \\
\hline Beam Divergence & $1 \mathrm{mrad}$ \\
\hline $\begin{array}{c}\text { Receiver } \\
\text { Dimension }\end{array}$ & $20 \mathrm{~cm}$ \\
\hline $\begin{array}{c}\text { Receiver sensitivity } \\
\text { Wavelength }\end{array}$ & $2000 \mathrm{nW}$ \\
\hline $\begin{array}{r}785 \mathrm{~nm}, 850 \mathrm{~nm}, 1550 \\
\mathrm{~nm}\end{array}$ \\
\hline
\end{tabular}

\section{Results and Discussion}

Fig. 4 demonstrates the relationship between BER and FSO visibility with various wavelengths. The possible minimum visibility that system can be achieved wills a better performance in an FSO link system. It is obviously evident that $1550 \mathrm{~nm}$ wavelength has the lowest BER at $10^{-16}$ and $2.6 \mathrm{~km}$ visibility. Meanwhile, at wavelength $850 \mathrm{~nm}$ and $785 \mathrm{~nm}$, the lowest BER are $10^{-6}$ and $10^{-4}$ at $4 \mathrm{~km}$ and $4.2 \mathrm{~km}$ visibility respectively. From the analysis that has been done, the $1550 \mathrm{~nm}$ wavelength shows the best performance regarding the lowest BER. Less signal power is loss along the transmission link when the BER is lower which directly improves the performance of the system. This means that higher wavelength system has better performance.

where: 


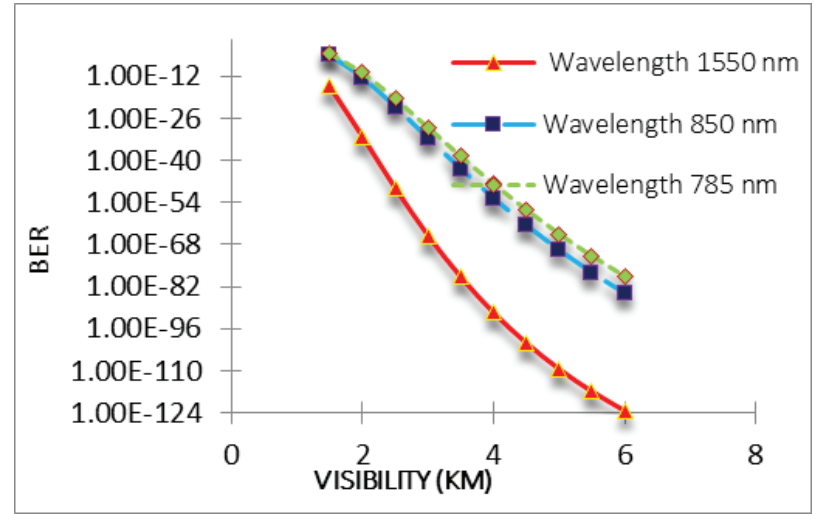

Fig. 4. Graph of visibility against bit error rate (BER) with adverse wavelength of $1550 \mathrm{~nm}, 850 \mathrm{~nm}$, and $785 \mathrm{~nm}$.

Fig. 5 demonstrates that maximum Q-factor keeps increasing as the visibility increases. The range of visibility studied in this paper is from $1.5 \mathrm{~km}$ to $6 \mathrm{~km}$ and the link range used is $1 \mathrm{~km}$. At visibility of $6 \mathrm{~km}$, the wavelength $1550 \mathrm{~nm}$ achieves the Q-factor of 24 compared to the wavelength of $785 \mathrm{~nm}$ and $850 \mathrm{~nm}$ as they are able to achieve the Q-factor of just 19 and 20 respectively. The wavelength of $1550 \mathrm{~nm}$ is chosen as the best wavelength to be used in the system when maximum Q-factor and visibility becomes higher. Thus the hybrid FSO system with wavelength $1550 \mathrm{~nm}$ could be improved by $21 \%$ compared to $5 \%$ improvement by wavelength $850 \mathrm{~nm}$ and $785 \mathrm{~nm}$.

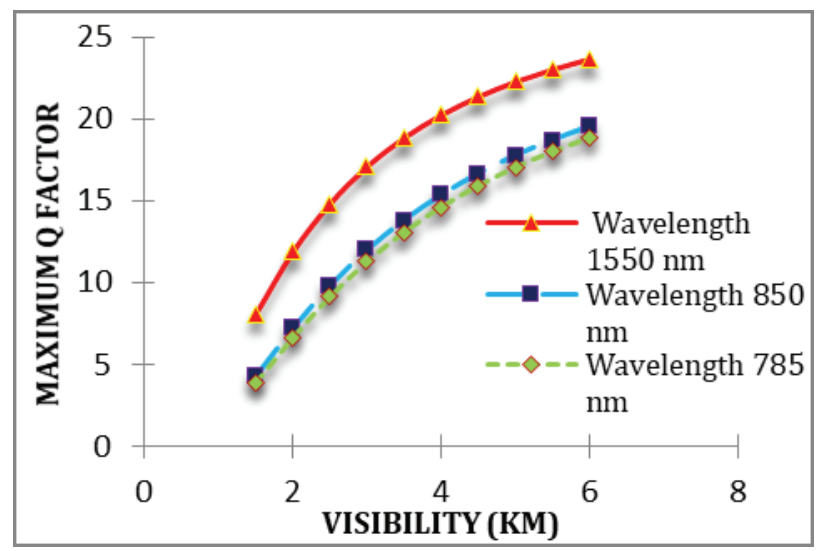

Fig. 5. Graph of visibility against maximum Q-factor with adverse wavelength of $1550 \mathrm{~nm}, 850 \mathrm{~nm}$, and $785 \mathrm{~nm}$.

The eye diagram is a useful tool for the qualitative analysis of signal used in digital transmission. Using wavelength $1550 \mathrm{~nm}$ in Fig. 6, the system has a large eye opening between the top and bottom level compared with wavelength $785 \mathrm{~nm}$, where the eye diagram shown in Fig. 7 is closer and smaller. The height of the eyeopening at the specified time interval shows the noise margin or noise immunity of the system. Based on these eye diagrams, it can be shown that $1550 \mathrm{~nm}$ wavelength produced a better performance compared to shorter wavelength because more error is produced due to lower value of Q-factor. As the value of Q factor increases, less signal power is loss. Thus promoting the link performance of FSO system. Another reason of using
$1550 \mathrm{~nm}$ is because of it is compatibility with current technology and device.

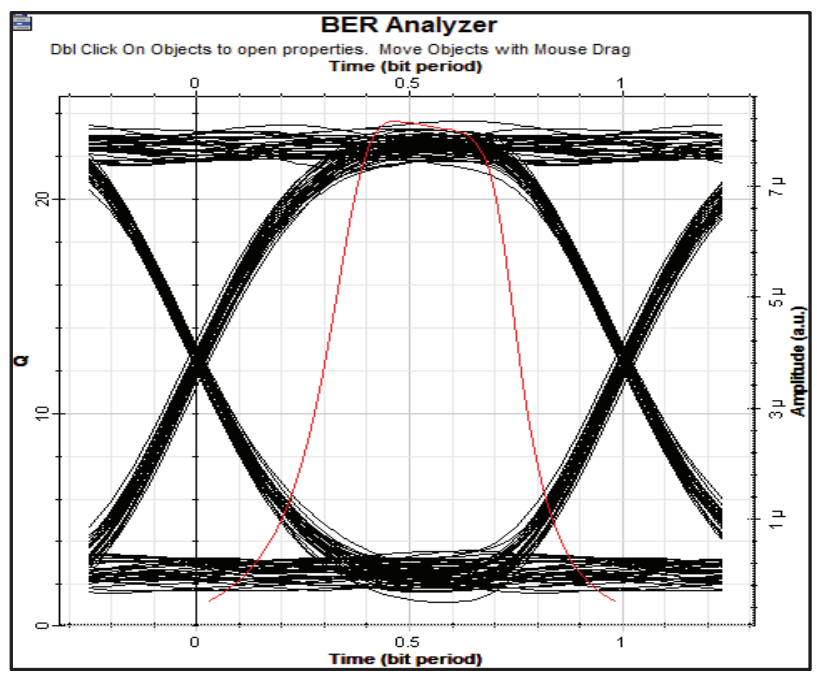

Fig. 6. Eye diagram of Q-factor for $1550 \mathrm{~nm}$ signal

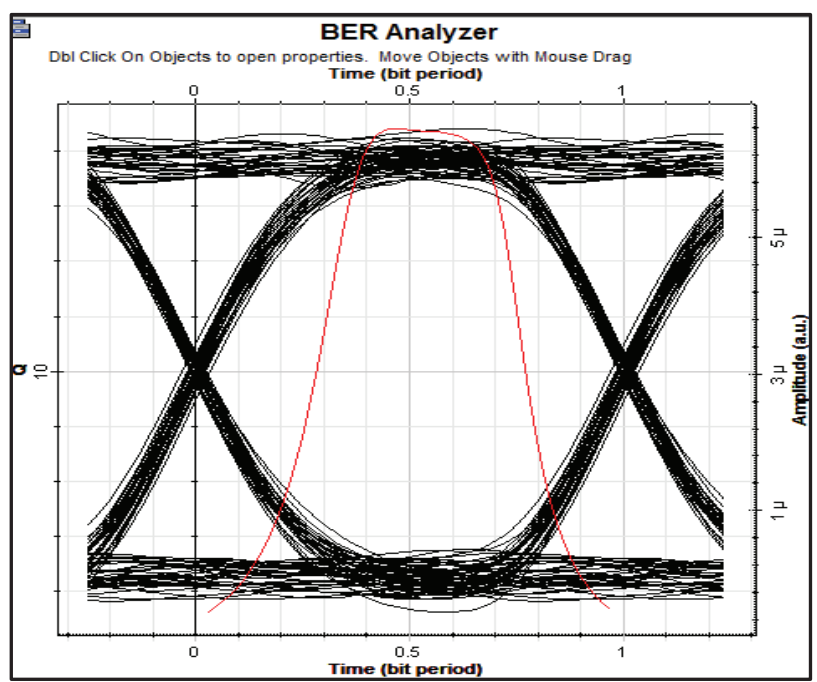

Fig. 7. Eye diagram of bit error rate for $1550 \mathrm{~nm}$ signal

\section{Conclusions}

The FSO systems reveal that communication system with wavelength $1550 \mathrm{~nm}$ at BER $10^{-124}$ is the best suited compared to the $850 \mathrm{~nm}$ and $785 \mathrm{~nm}$ wavelength. The result had shown that $1550 \mathrm{~nm}$ wavelength with low bit rate can produced optimum Q-factor of 25 . It can be concluded that, optical signal with higher bit rate is a superior choice to be used for higher output and less bit error rate. Moreover, the attenuation effect could also be reduced greatly.

This work was supported by the High Ministry of Education Malaysia under the Fundamental Research Grant Scheme (FRGS) \# 9003-000556 


\section{References}

1. A. Alkholidi, K.S. Altowij, Optical Communications Systems, 41-75 (2012)

2. H. Kaushal, G. Kaddoum, IEEE Communications Surveys \& Tutorials, 19, Issue 1 57-96 (2015)

3. Rashidi, C.B.M., Aljunid, S.A., Anuar, M.S., Fadhil, H.A., Ghani, F., Journal of Theoretical and Applied Information Technology. 61, Issue 1, Pages 155-159 (2014)

4. A.L. Puryear, Proc. SPIE, 7464, 74640J (2009)

5. P. Vishwakarma, J.P. IJAIEM, 2, 379-382 (2013)

6. Rashidi, C.B.M., Aljunid, S.A., Ghani, F., Fadhil, H.A., Anuar, M.S. Journal of Applied Sciences Research. 8, Issue 12, pp 5614-5626 (2012)

7. A.K. Rahman, S.A. Aljunid, M.S. Anuar, H.A. Fadhil, Key Engineering Materials, 594, 1027-1031 (2014)

8. X. Zhu, J. Kahn, IEEE Transactions on Communication, 50, 1293-1300 (2002)

9. A. Alkholidi, K.S. Altowij, Free Space Optical Communications - Theory and Practices, 159-212 (2014)

10. A.H. Hashim, Modelling Intersatellite Optical Wireless Communication System, Universiti Teknologi Malaysia, 1-65 (2009)

11. A.K. Rahman, S.A. Aljunid, M.S. Anuar, H.A. Fadhil, Journal of Theoretical and Applied Information Technology, 60, 1, 362-367 (2014)

12. K. Dev, R. Nebuloni, C. Capsoni, $10^{\text {th }}$ EuCAP on IEEE, 1-5 (2016)

13. Rashidi, C.B.M., Aljunid, S.A., Fadhil, H.A., Anuar, M.S. 4th International Conference on Photonics, Pages 166-168 (2013) 